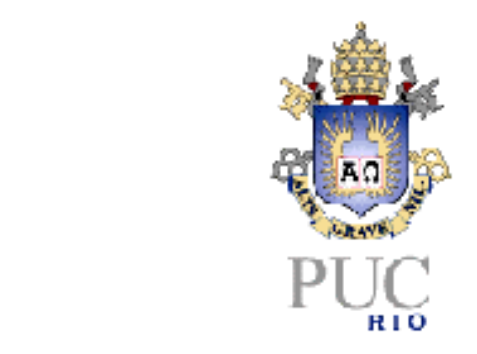

Rodrigo Flora Calili

Desenvolvimento de sistema para detecção de perdas comerciais em redes de distribuição de energia elétrica

\begin{abstract}
Dissertação de Mestrado
Dissertação apresentada ao Departamento de Engenharia Elétrica da PUC-Rio como parte dos requisitos para a obtenção do título de Mestre em Engenharia Elétrica da PUC-Rio.
\end{abstract}

Orientador: Reinaldo Castro Souza

Co-orientador: Henrique de O. Henriques

Rio de Janeiro Julho de 2005 


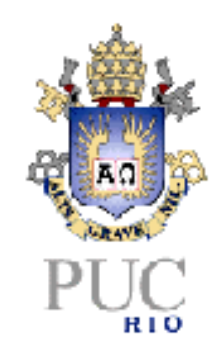

Rodrigo Flora Calili

\title{
Desenvolvimento de Sistema para Detecção \\ de Perdas Comerciais em Redes de Distribuição de Energia Elétrica
}

\begin{abstract}
Dissertação apresentada como requisito parcial para obtenção do grau de Mestre pelo Programa de PósGraduação em Engenharia Elétrica do Departamento de Engenharia Elétrica do Centro Técnico Científico da PUC-Rio. Aprovada pela Comissão Examinadora abaixo assinada.
\end{abstract}

Prof. Reinaldo Castro Souza

Orientador

Departamento de Engenharia Elétrica - PUC-Rio

Prof. Henrique de Oliveira Henriques

UFF

Prof. João Carlos de Oliveiras Aires

UGF/UFF

Prof. José Eugênio Leal

Coordenador Setorial do Centro

Técnico Científico - PUC-Rio

Rio de Janeiro, 28 de julho de 2005 
Todos os direitos reservados. É proibida a reprodução total ou parcial do trabalho sem autorização da universidade, do autor e do orientador.

\section{Rodrigo Flora Calili}

Graduou-se em Engenharia Elétrica na Universidade Federal de Juiz de Fora, Minas Gerais - Brasil no ano de 2003. Iniciou seus estudos de mestrado em 2003 no Departamento de Engenharia Elétrica da PUC-Rio.

Ficha catalográfica

Calili, Rodrigo Flora

Desenvolvimento de sistema para detecção de perdas comerciais em redes de distribuição de energia elétrica / Rodrigo Flora Calili ; orientador: Reinaldo Castro Souza ; coorientador: Henrique de O. Henriques. - Rio de Janeiro : PUCRio, Departamento de Engenharia Elétrica, 2005.

$157 \mathrm{f.} ; 30 \mathrm{~cm}$

Dissertação (mestrado) - Pontifícia Universidade Católica do Rio de Janeiro, Departamento de Engenharia Elétrica .

Inclui bibliografia

1. Engenharia Elétrica - Teses. 2. Adimplente. 3. Clusterização. 4. Fraudulento. 5. Inadimplente. 6. Perda comercial. I. Souza, Reinaldo Castro. II. Henriques, Henrique de O. III. Pontifícia Universidade Católica do Rio de Janeiro. Departamento de Engenharia Elétrica . IV. Título.

CDD: 621.3 
Este trabalho é inteiramente dedicado a minha amada mãe Marilene, a melhor mãe do mundo. 


\section{Agradecimentos}

Aos meus pais, Marilene e José Elias, pelo apoio incondicional em toda minha caminhada.

Aos meus irmãos, Hugo eLuiza, por seu apoio nas horas difíceis.

Ao meu avô Geraldo Flora que sempre torceu pelo meu sucesso.

A minha tia Eliana pela acolhida.

A todos meus familiares que, próximos ou distantes, me incentivaram nessa jornada.

A todos os amigos que, de alguma forma, contribuíram direta ou indiretamente pela realização do meu mestrado.

Ao meu orientador, professor doutor Reinaldo Souza Castro, pelo estímulo e auxílio para realização desta dissertação de mestrado.

Ao meu co-orientador, professor doutor Henrique Henriques da UFF, pela parceria na realização deste trabalho.

A CAPES e à PUC-Rio, pelos auxílios financeiros concedidos, sem os quais este trabalho não poderia ter sido realizado.

Aos colegas do NEC-PUC, especialmente ao Wesley, por todo auxílio com os bancos de dados.

Aos colegas Landi e João Carlos por toda ajuda concedida.

Aos colegas do Departamento de Engenharia Elétrica que de alguma forma contribuíram na confecção deste trabalho.

A Distribuidora de Energia ELEKTRO, especialmente Júlio Belan, que disponibilizou os dados utilizados neste trabalho.

Aos professores doutores que participaram da Comissão Examinadora. 


\section{Resumo}

Calili, Rodrigo Flora. Desenvolvimento de sistema para detecção de perdas comerciais em redes de distribuição de energia elétrica. Rio de Janeiro, 2005. 157p. Dissertação de Mestrado - Departamento de Engenharia Elétrica, Pontifícia Universidade Católica do Rio de Janeiro.

Os modelos matemáticos comumente usados na identificação de irregularidades na medição se baseiam na análise da redução percentual do consumo do mês (normalmente de $20 \%$ a $30 \%$ ) em relação aos meses anteriores. Este método tem gerado resultados imprecisos uma vez que considera o valor do consumo como um valor rígido e, portanto, não incorpora o efeito da sazonalidade na tipologia das cargas das unidades consumidoras.

Este trabalho tem o intuito de melhorar a identificação de clientes fraudulentos utilizando métodos de inteligência artificial, tais como Redes Neurais e Lógica Fuzzy, implementados a um banco de dados de cadastro da Distribuidora de Energia ELEKTRO e a uma Pesquisa de Posses e Hábitos de Consumo (PPH) feita nesta mesma empresa.

Nesta dissertação, o objetivo foi classificar um grupo de consumidores como normal (adimplente), inadimplente e fraudulento. Para tanto, foi feita inicialmente uma "clusterização" utilizando uma Rede Neural, mais especificamente uma Rede de Kohonen, para o banco de dados de cadastro disponibilizado pela distribuidora. Tomando os grupos desta classificação prévia feita pela Rede identificaram-se quais e quantos destes tiveram PPH's realizadas. Para se ter a classificação de um grupo quanto a incidência de consumidores normais, inadimplentes e fraudulentos utilizou-se um processo de Análise Fuzzy, o qual identifica os clusters com os consumidores de cada um dos segmentos. É feita uma análise de desempenho do modelo proposto com dados reais fornecidos pela empresa, na qual os resultados apontaram para uma robustez do método.

\section{Palavras chave:}

Adimplente, "Clusterização", Fraudulento, Inadimplente, Perda Comercial, PPH's. 


\section{Abstract}

Calili, Rodrigo Flora. System development to detect commercial losses in electrical energy distribution network. Rio de Janeiro, 2005. 157p. Master Dissertation - Electrical Engineering Department, Pontifícia Universidade Católica do Rio de Janeiro.

Mathematical models commonly used to identify irregularities in measurement are based on percentile reduction analysis of the monthly consumption (normally from $20 \%$ to $30 \%$ ) in relation to the previous months. This method tends to generate imprecise results, since it considers the value of the consumption as a rigid value and, therefore, it does not incorporate the seasonal effect in the loads topology of the consumer units.

This work has intention to improve the identification of fraudulent customers using artificial intelligence methods, such as Neural Networks and Fuzzy Logic, implemented to a database of consumers of ELEKTRO a distributing utility of São Paulo State, Brazil. It also uses information on appliances ownership obtained via market research in ELEKTRO area, named PPH (Portuguese for this particular type of market research).

In this dissertation, the main objective was to classify a group of consumers as solvent, insolvent and fraudulent. In order to achieve this task, a clustering was initially made using a Neural Network framework, more specifically a Kohonen Network, for the database available. It was then checked which of the groups had a minimum number of clients interviewed in the PPH. In order to have the classification of the clients in the three categories it was used Fuzzy Analysis. Selected data is also presented, considering the available database of the Company as well as the research environment, which had been taken from the PPH. Finally, it was checked the performance of the method against real data obtained from the utility and the results were very satisfactory.

\section{Keywords:}

Clustering, Fraudulent, Commercial Loss, Insolvent, PPH's, Solvent. 


\section{SUMÁRIO}

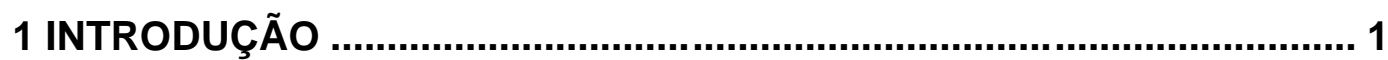

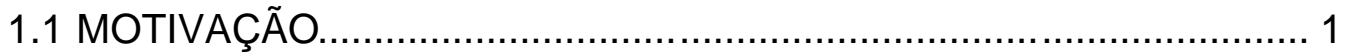

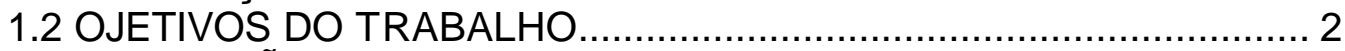

1.3 DESCRIÇÃO DO TRABALHO ................................................. 4

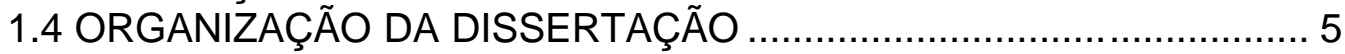

2 PERDAS DE ENERGIA ...................................................................... 8

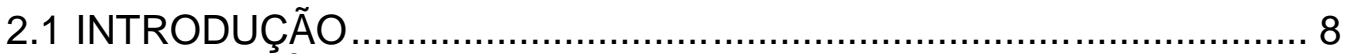

2.2 PERDAS TÉCNICAS .............................................................. 10

2.2.1 Perdas Técnicas no Sistema de Transmissão ............................ 11

2.2.1.1 Medição ...................................................................... 11

2.2.1.2 Load Flow ................................................................... 11

2.2.2 Perdas Técnicas no Sistema de Distribuição ............................. 11

2.2.2.1 Modelos Simplificados .................................................... 11

2.2.2.2 Fluxo de Potência .............................................................. 12

2.3 PERDAS COMERCIAIS .......................................................... 13

2.3.1 Introdução....................................................................... 13

2.3.2 A Perda Comercial e a Inadimplência ........................................ 14

2.3.3 A Perda Comercial e a Qualidade de Atendimento aos Clientes. 16

2.3.4 A Perda Comercial e o Medidor de Energia Elétrica ................... 18

2.3.5 A Perda Comercial e a Violência............................................. 20

2.3.6 A Perda Comercial e a Economia Brasileira .................................. 23

2.3.7 A Perda Comercial e o Racionamento ...................................... 25

2.3.8 A Perda Comercial e a Revisão Tarifária ................................. 26

3 FERRAMENTAS UTILIZADAS: REDES NEURAIS E LÓGICA FUZZY.. 27

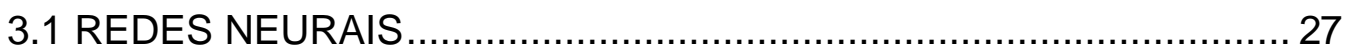

3.2 MAPA AUTO-ORGANIZÁVEL DE KOHONEN ............................. 29

3.2.1 Introdução.................................................................. 29

3.2.2 A Rede de Kohonen ........................................................ 31

3.2.3 Processo de Aprendizagem................................................ 33

3.2.4 Algoritmo de Kohonen ........................................................ 34

3.3 LÓGICA FUZZY ................................................................ 36

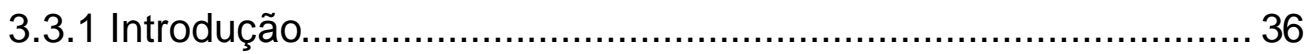

3.3.2 Conjuntos Fuzzy ........................................................... 38

3.3.3 Conectivos Lógicos .......................................................... 40

3.3.4 Proposições ou Regras Fuzzy ........................................... 41

3.3.5 Inferência Fuzzy ................................................................ 42

3.3.6 Defuzzificação .............................................................. 42 
4 DESCRIÇÃO DO MODELO DE DETECÇÃO DE PERDAS

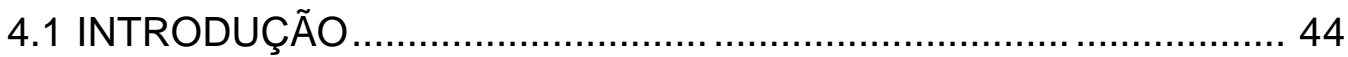

4.2 DADOS SELECIONADOS ………................................................. 45

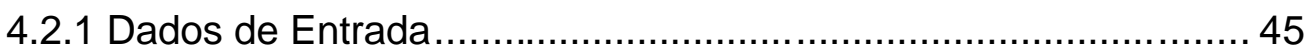

4.2.2 Dados de pesquisa........................................................... 49

4.2.3 Processo de Ligação dos Dados de Entrada com os Dados de

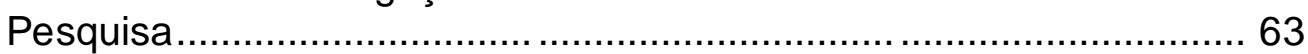

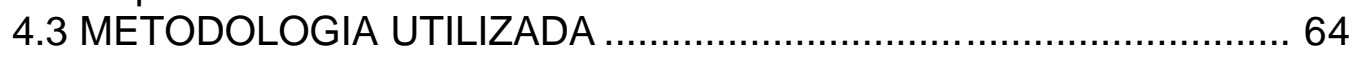

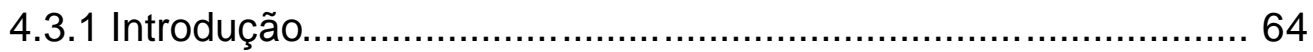

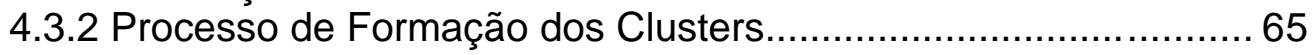

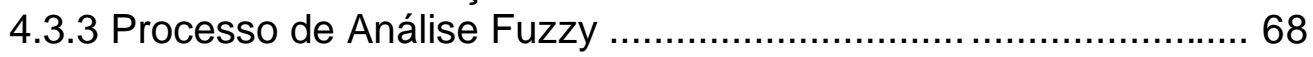

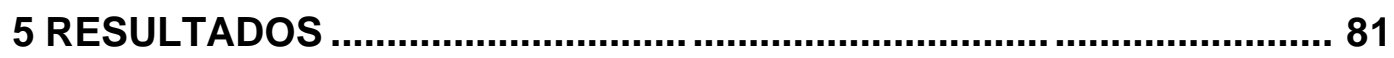

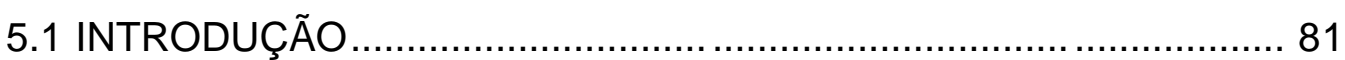

5.2 ANÁLISE PARA METADE DOS CLIENTES DE GUARUJÁ …............82

5.2.1 Classificação por Rede de Kohonen ........................................ 82

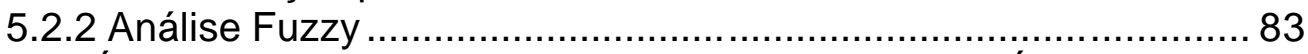

5.3 ANÁLISE PARA TODOS OS CLIENTES DE GUARUJÁ.................... 87

5.3.1 Classificação por Rede de Kohonen ....................................... 87

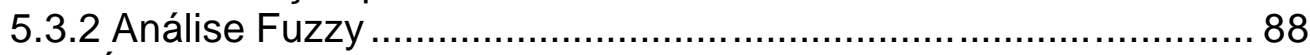

5.4 ANÁLISE FINAL DOS RESULTADOS …………........................ 92

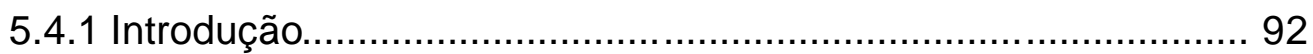

5.4.2 Comparação entre Metade e Todos os Clientes de Guarujá ....... 93

5.4.2.1 Clientes Fraudulentos ..................................................... 93

5.4.2.2 Clientes Inadimplentes.................................................. 94

5.4.3 Procura dos Dados Reais nos Clusters Gerados ...................... 95

5.4.3.1 Clientes Fraudulentos ......................................................... 96

5.4.3.2 Clientes Inadimplentes.................................................... 97

6 CONCLUSÕES E TRABALHOS FUTUROS …….................................. 99

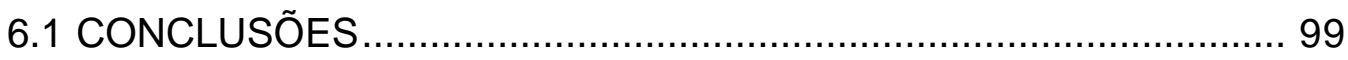

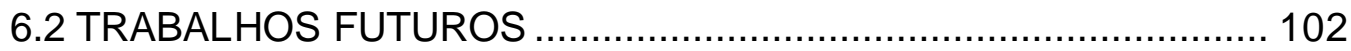

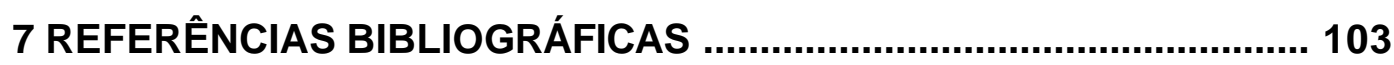

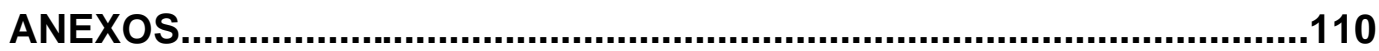




\section{Lista de llustrações}

Figura 1: Evolução do preço das tarifas médias de energia e do salário mínimo real...............................................................................15

Figura 2: Satisfação dos clientes da ELEKTRO............................................17

Figura 3: Nível de escolaridade dos clientes da ELEKTRO.........................25

Figura 4: Representação de uma rede de Kohonen....................................33

Figura 5: Comparação entre um conjunto crisp e um conjunto fuzzy............39

Figura 6: Diferentes variáveis lingüísticas para a variável temperatura

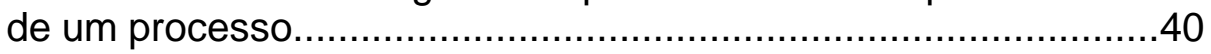

Figura 7: Caracterização de um cliente na variável média de consumo........48

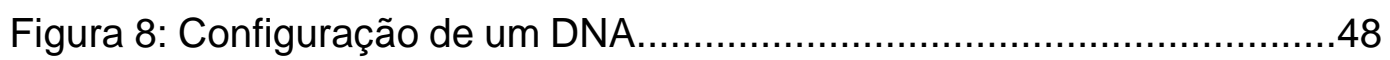

Figura 9: Número de moradores em cada domicílio entrevistado..................50

Figura 10: Tempo que o entrevistado mora no domicílio...............................51

Figura 11: Área construída do domicílio em $\mathrm{m}^{2}$.........................................51

Figura 12: Posse média de lâmpadas.......................................................52

Figura 13: Posse média de eletrodomésticos (1)..........................................53

Figura 14: Posse média de eletrodomésticos (2)......................................54

Figura 15: Renda familiar declarada pelo entrevistado (em s.m.).................58

Figura 16: Classificação da região do domicílio pelo pesquisador................59

Figura 17: Proximidade do domicílio em relação a favela..............................60

Figura 18: Peso da conta de luz no orçamento do entrevistado....................60

Figura 19: Esquema de ligação entre os dados de entrada e dados de pesquisa pela Uc.

Figura 20: Esquema de formação de clusters .66

Figura 21: Curva de freqüência normalizada das respostas para cada uma das 40 variáveis. 
Figura 22: Curva de freqüência respostas para os clientes normais com o corte.

Figura 23: Curva de freqüência respostas para os clientes fraudulentos com o corte.

Figura 24: Curva de freqüência respostas para os clientes inadimplentes com o corte.

Figura 25: Curva de pertinência para variável 9 dos clientes normais..........75

Figura 26: Curva de pertinência para variável 31 dos clientes fraudulentos.

Figura 27: Curvas de pertinê ncia das respostas de clientes adimplentes às 8 perguntas selecionadas.

Figura 28: Curvas de pertinência das respostas de clientes fraudulentos às 13 perguntas selecionadas.

Figura 29: Curvas de pertinência das respostas de clientes inadimplentes às 14 perguntas selecionadas

Figura 30: Resultado final da classificação fuzzy dos clusters.

Figura 31: Curvas de pertinência de clientes adimple ntes para metade de Guarujá......

Figura 32: Curvas de pertinência de clientes fraudulentos para metade de Guarujá

Figura 33: Curvas de pertinência de clientes inadimplentes para metade de Guarujá

Figura 34: Resultado final da classificação fuzzy dos clusters para metade de Guarujá.

Figura 35: Curvas de pertinência de clientes adimple ntes de Guarujá.........89

Figura 36: Curvas de pertinência de clientes fraudulentos de Guarujá........89

Figura 37: Curvas de pertinência de clientes inadimplentes de Guarujá......90

Figura 38: Resultado final da classificação fuzzy dos clusters para metade de Guarujá 


\section{Lista de tabelas}

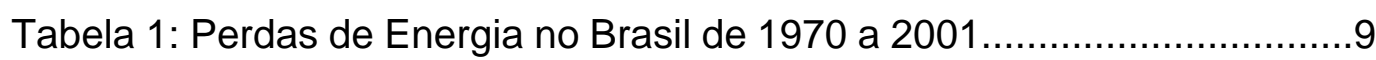

Tabela 2: Teste -t para a posse média de eletrodomésticos........................56

Tabela 3: Teste de Levene para a posse média de eletrodomésticos.........56

Tabela 4: Relação das variáveis selecionadas no questionário da PPH.....61

Tabela 5: Relação das variáveis selecionadas e suas respectivas faixas....62

Tabela 6: Pertinência para as variáveis 9 dos clientes normais e 31 dos clientes fraudulentos.............................................................. 74

Tabela 7: Número de consumidores e clientes pesquisados por cluster (metade de Guarujá) ..............................................................83

Tabela 8: Número de consumidores e clientes pesquisados por cluster (Guarujá)

Tabela 9: Comparação entre os clientes fraudulentos de metade e toda Guarujá.

Tabela 10: Comparação entre os clientes inadimplentes de metade e toda Guarujá.

Tabela 11: Verificação dos clientes fraudulentos reais encontrados nos clusters de Guarujá.

Tabela 12: Verificação dos clientes inadimplentes reais encontrados nos clusters de Guarujá. 